\title{
Non-abelian Wilson surfaces
}

\author{
Iouri Chepelev \\ Department of Physics, California Institute of Technology \\ Pasadena, CA 91125, USA, and \\ C.N. Yang Institute for Theoretical Physics \\ SUNY at Stony Brook, NY 11794, USA \\ Email: chepelev@theory.caltech.edu
}

ABSTRACT: A definition of non-abelian genus zero open Wilson surfaces is proposed. The ambiguity in surface-ordering is compensated by the gauge transformations.

KeYwords: p-branes, Gauge Symmetry, Differential and Algebraic Geometry. 


\section{Contents}

1. Introduction 1

2. Definition 2

3. Gauge transformations 国

4. Comments 8

\section{Introduction}

A higher dimensional generalization of the non-abelian Wilson line is not known. Only recently the notion of a connection on a non-abelian 1-gerbe was introduced in the work of Breen and Messing [1].

A motivation for defining the Non-abelian Wilson Surfaces comes from the string theory. NWS are relevant to six dimensional theories on the world volumes of coincident five branes [2].

The main problem in defining NWS is the lack of a natural order on a 2-dimensional surface. A naive guess for the NWS is

$$
P \exp \left(\int_{\Sigma} B\right)
$$

where $B$ is a non-abelian 2-form. The choice of a surface-ordering $P$ involves a time-slicing of the 2-surface $\Sigma$. A no-go theorem of Teitelboim [3 states that no such a choice is compatible with the reparametrization invariance.

Let us recall the notion of a connection on a non-abelian 1-gerbe [1]. A connection on a principal bundle (0-gerbe) can be thought of as follows. Let $x_{0}$ and $x_{1}$ be two infinitesimally close points. The fibers $S_{x_{0}}$ and $S_{x_{1}}$ over these points are sets and the connection is a function

$$
f_{01}: S_{x_{1}} \rightarrow S_{x_{0}} .
$$

The connection on a non-abelian 1-gerbe is defined by analogy with the 0-gerbe case 囵. The fibers are categories $C_{x_{0}}$ and $C_{x_{1}}$, and the connection is a functor

$$
\varepsilon_{01}: C_{x_{1}} \rightarrow C_{x_{0}} \text {. }
$$

Let $x_{0}, x_{1}$ and $x_{2}$ be three infinitesimally close points. A diagram of functors and natural transformations is shown in figure 1. Let $\operatorname{Aut}(G)$ be the group of automorphisms of a non-abelian group $G$. Let $\operatorname{Lie}(G)$ be the Lie algebra of $G$. It is shown in [1] that 2-arrow $K, 1$-arrow $\kappa$ and 1-arrow $\varepsilon$ in the diagram correspond to a $\operatorname{Lie}(G)$-valued 2-form $B$, a $\operatorname{Lie}(\operatorname{Aut}(G))$-valued 2-form $\nu$ and a $\operatorname{Lie}(\operatorname{Aut}(G))$-valued 1-form $\mu$ respectively.

The paper is organized as follows. In section 2 a definition of NWS is proposed. Section 3 is devoted to gauge transformations. Some comments are listed in section 4. 


\section{Definition}

We interpret the infinitesimal 2-simplex in figure 1 as a transmuted form of an infinitesimal Wilson surface expressed in the language of category theory. The fibered category in the formulation of [1] can be thought of as an 'internal symmetry space' of a non-abelian string. Let $\Sigma$ be a 2 -dimensional surface with the disk topology. Let $C$ be a clockwise oriented boundary of $\Sigma$ and $P$ a marked point on it (see figure 2). We associate group elements

$$
W[\Sigma, C, P] \in G
$$

and

$$
V[\Sigma, C, P] \in \operatorname{Aut}(G)
$$

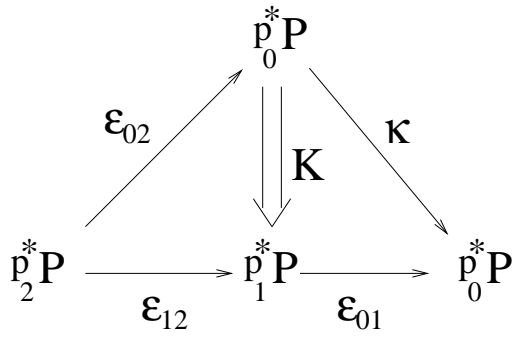

Figure 1: $\varepsilon_{i j}$ is a cartesian functor from the fibered category $p_{j}^{*} P$ to $p_{i}^{*} P, \kappa$ is a cartesian functor from $p_{0}^{*} P$ to $p_{0}^{*} P$, and $K$ is a 2 arrow from $\kappa \circ \varepsilon_{02}$ to $\varepsilon_{01} \circ \varepsilon_{12}$.

with the data $(\Sigma, C, P)$. We write $W[\Sigma]$ and $V[\Sigma]$ when the omitted arguments are obvious from the context. With an open curve $C$ we associate an element of $\operatorname{Aut}(G)$ :

$$
M[C] \in \operatorname{Aut}(G) .
$$

Let $C=C_{2} \circ C_{1}$ be a composition of curves $C_{2}$ and $C_{1}$. We assume

$$
M[C]=M\left[C_{2} \circ C_{1}\right]=M\left[C_{2}\right] M\left[C_{1}\right] .
$$

We now propose an equation relating $M[C], W[\Sigma, C]$ and $V[\Sigma, C]$. For a group element $g \in G$ we denote by $i_{g}$ the inner automorphism

$$
i_{g}(h)=g h g^{-1}, \quad \forall h \in G .
$$

The conjectural equation reads

$$
M[C]=i_{W[\Sigma]} V[\Sigma] .
$$

An infinitesimal version of this equation was first derived in [1] from the requirement that $K$ in figure 1 is a natural transformation. We regard eq. (2.4) as a fundamental equation relating bulk and boundary of the non-abelian string world-sheet.

Eq. (2.4) can be used to find a composition rule for two NWS. Consider the 2-surfaces in figure 2. The identity

$$
\begin{aligned}
i_{W\left[\Sigma_{2} \circ \Sigma_{1}, P_{1}\right]} V\left[\Sigma_{2} \circ \Sigma_{1}\right] & =M\left[C \circ C_{4} \circ C_{3}\right] \\
& =M[C] M\left[C_{4} \circ C_{5}^{-1}\right] M\left[C^{-1}\right] M\left[C \circ C_{5} \circ C_{3}\right] \\
& =M[C] i_{W\left[\Sigma_{2}, P_{2}\right]} V\left[\Sigma_{2}, P_{2}\right] M\left[C^{-1}\right] i_{W\left[\Sigma_{1}, P_{1}\right]} V\left[\Sigma_{1}, P_{1}\right]
\end{aligned}
$$

suggests the following composition rule for Wilson surfaces:

$$
\begin{aligned}
W\left[\Sigma_{2} \circ \Sigma_{1}\right] & =M[C]\left(W\left[\Sigma_{2}\right]\right) M[C] V\left[\Sigma_{2}\right] M\left[C^{-1}\right]\left(W\left[\Sigma_{1}\right]\right), \\
V\left[\Sigma_{2} \circ \Sigma_{1}\right] & =M[C] V\left[\Sigma_{2}\right] M\left[C^{-1}\right] V\left[\Sigma_{1}\right] .
\end{aligned}
$$



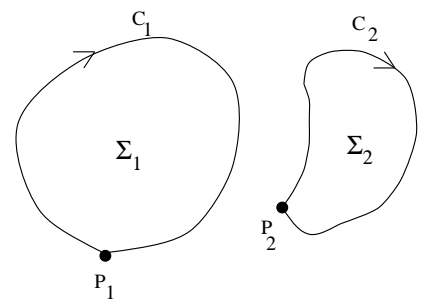

(a)

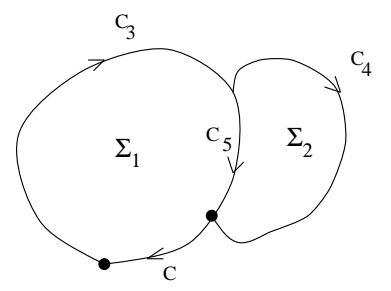

(b)

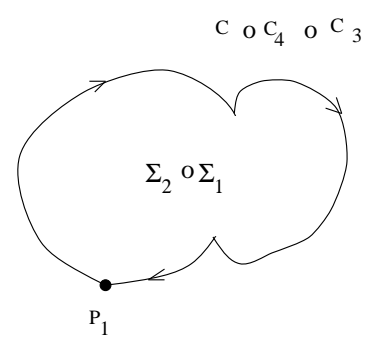

(c)

Figure 2: Composition of surfaces with the disk topology. (a) Surfaces $\Sigma_{i}$ with the marked points $P_{i}$ and the clockwise oriented boundaries $C_{i}$. (b) Surfaces are joined along the common boundary segment $C_{5}$. (c) The resulting surface $\Sigma_{2} \circ \Sigma_{1}$ with the marked point $P_{1}$ and the clockwise oriented boundary $C \circ C_{4} \circ C_{3}$.

An infinitesimal version of eq. (2.6) appeared implicitly in the category-theoretic definition of the curvature in [1].

Eq. (2.6) can be understood as follows. When the curve $C$ is absent, i.e. when the marked points of $\Sigma_{1}$ and $\Sigma_{2}$ coincide, eq. (2.6) simplifies to

$$
\begin{aligned}
W\left[\Sigma_{2} \circ \Sigma_{1}\right] & =W\left[\Sigma_{2}\right] V\left[\Sigma_{2}\right]\left(W\left[\Sigma_{1}\right]\right), \\
V\left[\Sigma_{2} \circ \Sigma_{1}\right] & =V\left[\Sigma_{2}\right] V\left[\Sigma_{1}\right] .
\end{aligned}
$$

Thus when the marked points of the two surfaces coincide, the Wilson surfaces are composed as in eq. (2.7). If we think of $V[\Sigma, P]$ as an operator which acts on the objects with the marked point $P$ and assume that only the objects with the same marked points can be multiplied, then the meaning of eq. (2.6) becomes clear. The role of $M[C]$ in eq. (2.6) is to transform the objects with the marked point $P_{2}$ to the objects with the marked point $P_{1}$.

Composition of three or more surfaces is in general ambiguous. Consider figure 3. Using the composition rule (2.6) it can be shown that

$$
\begin{aligned}
W\left[\Sigma_{3} \circ\left(\Sigma_{2} \circ \Sigma_{1}\right)\right] & \neq W\left[\Sigma_{2} \circ\left(\Sigma_{3} \circ \Sigma_{1}\right)\right], \\
V\left[\Sigma_{3} \circ\left(\Sigma_{2} \circ \Sigma_{1}\right)\right] & \neq V\left[\Sigma_{2} \circ\left(\Sigma_{3} \circ \Sigma_{1}\right)\right] .
\end{aligned}
$$

Given

$$
V[\delta \Sigma] \approx 1+v[P] \equiv 1+v_{\mu \nu}[P] \sigma^{\mu \nu}
$$

for an infinitesimal surface $\delta \Sigma$ with the area element $\sigma^{\mu \nu}$, we want to find $V[\Sigma]$ for a finitesize surface $\Sigma$. This can be done using a trick similar to the one used in the context of the non-abelian Stokes formula [4]. Consider the contour $C^{\prime}$ in figure 4 . From the relation

$$
M\left[C^{\prime}\right]=M\left[C_{P}^{-1}\right] M[\delta C] M\left[C_{P}\right] M[C]
$$




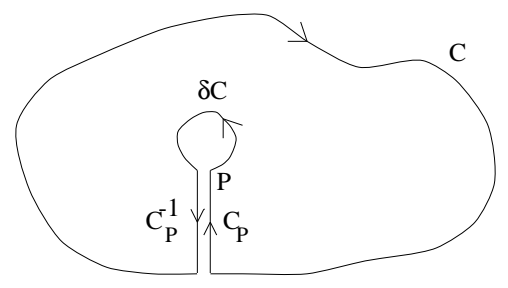

Figure 4: Contour $C^{\prime}=C_{P}^{-1} \circ \delta C \circ C_{P} \circ C$.

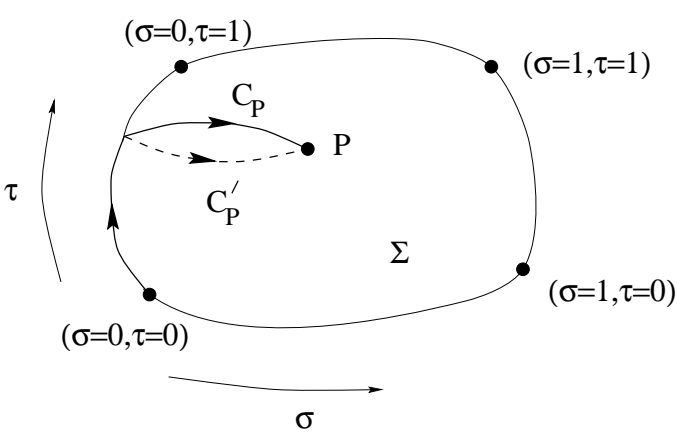

Figure 5: A parametrized surface $\Sigma$. The path $C_{P}$ consists of two segments: the first segment ( $\sigma=0=$ const., $\tau$ ) is from $\tau=0$ to $\tau$ and the second segment ( $\sigma, \tau=$ const.) is from $\sigma=0$ to $\sigma$.

and eq. (2.4) one finds

$$
V\left[\Sigma^{\prime}\right]=M\left[C_{P}^{-1}\right] V^{-1}[\delta \Sigma] M\left[C_{P}\right] V[\Sigma] .
$$

Thus we have

$$
\delta V[\Sigma]=M\left[C_{P}^{-1}\right] v[P] M\left[C_{P}\right] V[\Sigma] .
$$

A solution of this equation involves a choice of ordering and it is given by

$$
V[\Sigma]=\hat{P}_{\tau} \exp \left(\int_{\Sigma} M\left[C_{P}^{-1}\right] v[P] M\left[C_{P}\right]\right),
$$

where $\hat{P}_{\tau}$ is the ordering in $\tau$ and the curve $C_{P}$ is defined in figure 5 . Note that the expression eq. (2.13) depends on the parametrization $x^{\mu}=x^{\mu}(\sigma, \tau)$ of the surface $\Sigma$. For example a boundary-preserving reparametrization will change $C_{P}$ to a $C_{P}^{\prime}$ (see figure 5). Thus $V[\Sigma]$ and $W[\Sigma]$ depend on the parametrization of $\Sigma$ :

$$
V=V\left[\Sigma, x^{\mu}(\sigma, \tau)\right], \quad W=W\left[\Sigma, x^{\mu}(\sigma, \tau)\right] .
$$

In section 3 we will see that if $(\sigma, \tau)$ and $(\tilde{\sigma}, \tilde{\tau})$ are two different parametrizations of a surface $\Sigma$, then

$$
\left(V\left[\Sigma, x^{\mu}(\sigma, \tau)\right], W\left[\Sigma, x^{\mu}(\sigma, \tau)\right]\right)
$$

and

$$
\left(V\left[\Sigma, x^{\mu}(\tilde{\sigma}, \tilde{\tau})\right], W\left[\Sigma, x^{\mu}(\tilde{\sigma}, \tilde{\tau})\right]\right)
$$

are related by the gauge transformation. In other words, the non-abelian internal symmetry and the reparametrization symmetry mix.

\section{Gauge transformations}

In this section we introduce the gauge transformations which compensate the ambiguity in the composition of NWS. Suppose that a surface $\Sigma$ is composed out of three or more smaller 
surfaces. Let $(W[\Sigma], V[\Sigma])$ and $(\tilde{W}[\Sigma], \tilde{V}[\Sigma])$ correspond to two different compositions resulting in the surface $\Sigma$. We have

$$
M[C]=i_{W[\Sigma]} V[\Sigma]=i_{\tilde{W}[\Sigma]} \tilde{V}[\Sigma] .
$$

Since $W$ and $\tilde{W}$ are elements of a group $G$, there is a group element $R[\Sigma] \in G$ such that

$$
\tilde{W}[\Sigma]=W[\Sigma](R[\Sigma])^{-1} .
$$

Let us decompose $W$ and $\tilde{W}$ into the abelian and non-abelian factors:

$$
W=W_{\mathrm{ab}} \cdot W_{\text {nonab }}, \quad \tilde{W}=\tilde{W}_{\mathrm{ab}} \cdot \tilde{W}_{\text {nonab }} .
$$

It is clear that the ambiguity in the composition does not affect the abelian part. Thus we have

$$
\tilde{W}_{\mathrm{ab}}[\Sigma]=W_{\mathrm{ab}}[\Sigma] .
$$

Combining this equation with eq. (3.2) we find

$$
\tilde{W}_{\text {nonab }}[\Sigma]=W_{\text {nonab }}[\Sigma](R[\Sigma])^{-1} .
$$

We propose that eq. (3.4) and eq. (3.5) define the gauge transformation of $W$. In order for this gauge transformation of $W$ to be compatible with eq. (3.1), $V$ should transform as

$$
\tilde{V}[\Sigma]=i_{R[\Sigma]} V[\Sigma] .
$$

It can be checked that the gauge transformations (3.4)-(3.6) are compatible with the composition rule (2.6) provided that the composition rule for $R$ is the same as that of $W$, namely

$$
R\left[\Sigma_{2} \circ \Sigma_{1}\right]=M[C]\left(R\left[\Sigma_{2}\right]\right) M[C] V\left[\Sigma_{2}\right] M\left[C^{-1}\right]\left(R\left[\Sigma_{1}\right]\right) .
$$

More generally, consider a surface $\Sigma$ divided into $n$ smaller surfaces $\Sigma_{1}, \ldots, \Sigma_{n}$. Let $C$ be the boundary of $\Sigma$. Repeating the reasoning leading to eq. (2.6) we have

$$
M[C]=M\left[\mathcal{C}_{1}\right] i_{W\left[\Sigma_{1}\right]} V\left[\Sigma_{1}\right] M\left[\mathcal{C}_{2}\right] i_{W\left[\Sigma_{2}\right]} V\left[\Sigma_{2}\right] M\left[\mathcal{C}_{3}\right] \cdots
$$

for some curves $\mathcal{C}_{1}, \mathcal{C}_{2}, \ldots$. From this equation we find

$$
\begin{aligned}
W[\Sigma] & =M\left[\mathcal{C}_{1}\right]\left(W\left[\Sigma_{1}\right]\right) M\left[\mathcal{C}_{1}\right] V\left[\Sigma_{1}\right] M\left[\mathcal{C}_{2}\right]\left(W\left[\Sigma_{2}\right]\right) \cdots \\
V[\Sigma] & =M\left[\mathcal{C}_{1}\right] V\left[\Sigma_{1}\right] M\left[\mathcal{C}_{2}\right] V\left[\Sigma_{2}\right] M\left[\mathcal{C}_{3}\right] \cdots
\end{aligned}
$$

It is easy to see that the gauge transformations (3.4) $-(3.6)$ are compatible with eq. (3.9) provided that $R[\Sigma]$ is composed out of $R\left[\Sigma_{i}\right]$ as follows:

$$
R[\Sigma]=M\left[\mathcal{C}_{1}\right]\left(R\left[\Sigma_{1}\right]\right) M\left[\mathcal{C}_{1}\right] V\left[\Sigma_{1}\right] M\left[\mathcal{C}_{2}\right]\left(R\left[\Sigma_{2}\right]\right) \cdots .
$$

Thus $R$ should be composed by the rule of composition of $W$.

We now introduce new gauge transformations. These are the transformations of $M$, $V$ and $W$ compatible with eq. (2.4). 
Let $\Lambda[P]$ be an $\operatorname{Aut}(G)$-valued function of point $P$. Let $C$ be a directed path from $P_{1}$ to $P_{2}$. The gauge transformation of $M[C]$ reads

$$
\tilde{M}[C]=\Lambda\left[P_{2}\right] M[C] \Lambda\left[P_{1}\right]^{-1} .
$$

When $P_{1}=P_{2}=P$ this equation becomes

$$
\tilde{M}[C]=\Lambda[P] M[C] \Lambda[P]^{-1} .
$$

From this equation and

$$
\tilde{M}[C]=i_{\tilde{W}} \tilde{V}
$$

one finds

$$
i_{W} V=\Lambda^{-1} i_{\tilde{W}} \tilde{V} \Lambda=i_{\Lambda^{-1}(\tilde{W})} \Lambda^{-1} \tilde{V} \Lambda
$$

Thus we propose the gauge transformations:

$$
\begin{aligned}
\tilde{V}[\Sigma, P] & =\Lambda[P] V[\Sigma, P] \Lambda[P]^{-1}, \\
\tilde{W}[\Sigma, P] & =\Lambda[P](W[\Sigma, P]) .
\end{aligned}
$$

We now consider a new gauge transformation which is a finite generalization of the infinitesimal transformation considered in [1]. The transformation reads

$$
\tilde{M}[C]=i_{\mathcal{Z}[C]} M[C],
$$

where $\mathcal{Z}[C]$ is a $G$-valued functional of $C$. The composition rule for $\mathcal{Z}$ can be inferred from the following chain of equations:

$$
\begin{aligned}
i_{\mathcal{Z}\left[C_{2} \circ C_{1}\right]} M\left[C_{2} \circ C_{1}\right] & =\tilde{M}\left[C_{2} \circ C_{1}\right] \\
& =\tilde{M}\left[C_{2}\right] \tilde{M}\left[C_{1}\right] \\
& =i_{\mathcal{Z}\left[C_{2}\right]} M\left[C_{2}\right] i_{\mathcal{Z}\left[C_{1}\right]} M\left[C_{1}\right] \\
& =i_{\mathcal{Z}\left[C_{2}\right]} i_{M\left[C_{2}\right]\left(\mathcal{Z}\left[C_{1}\right]\right)} M\left[C_{2} \circ C_{1}\right] .
\end{aligned}
$$

This equation suggests the following composition rule for $\mathcal{Z}$ :

$$
\mathcal{Z}\left[C_{2} \circ C_{1}\right]=\mathcal{Z}\left[C_{2}\right] M\left[C_{2}\right]\left(\mathcal{Z}\left[C_{1}\right]\right) .
$$

If a $\operatorname{Lie}(G)$-valued 1-form $\zeta$ is given, $\mathcal{Z}[C]$ for an open path $C$ can be constructed as follows. Let us divide $C$ into $n$ small subpaths as in figure 6 a. Applying eq. (3.18) we find

$$
\begin{aligned}
\mathcal{Z}[C]= & \mathcal{Z}\left[C_{n}\right] \cdot M\left[C_{n}\right]\left(Z\left[C_{n-1}\right]\right) \cdot M\left[C_{n} \circ C_{n-1}\right]\left(\mathcal{Z}\left[C_{n-2}\right]\right) \times \cdots \\
& \times M\left[C_{n} \circ C_{n-1} \cdots C_{2}\right]\left(\mathcal{Z}\left[C_{1}\right]\right) \\
\approx & \left(1+\zeta_{\mu}\left[P_{n}\right] d x^{\mu}\right)\left(1+M\left[C_{n}\right]\left(\zeta_{\mu}\left[P_{n-1}\right]\right) d x^{\mu}\right) \times \cdots \\
& \times\left(1+M\left[C_{n} \circ C_{n-1} \cdots C_{2}\right]\left(\zeta_{\mu}\left[P_{1}\right]\right) d x^{\mu}\right) .
\end{aligned}
$$

In the large $n$ limit we thus find

$$
\mathcal{Z}[C]=\hat{P} \exp \left(\int_{C} M\left[C^{\prime \prime}\right]\left(\zeta_{\mu}[P]\right) d x^{\mu}\right)
$$




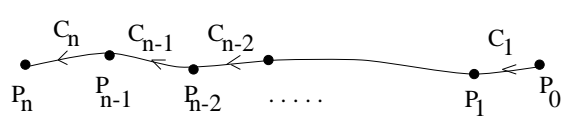

(a)

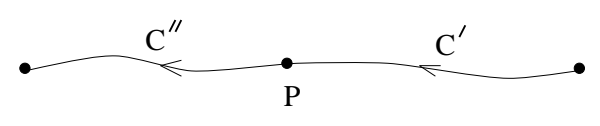

(b)

Figure 6: $(a)$ The path $C$ is divided into $n$ small subpaths: $C=C_{n} \circ C_{n-1} \cdots \circ C_{1}$. (b) The point $P$ divides $C=C^{\prime \prime} \circ C^{\prime}$.

where $C^{\prime \prime}$ and $P$ are as in figure $6 \beta$, and $\hat{P}$ is the path ordering operator.

A choice of transformation of $V$ and $W$ compatible with eq. (2.4) and eq. (3.16) is

$$
\begin{aligned}
\tilde{V}[\Sigma, C] & =V[\Sigma, C], \\
\tilde{W}[\Sigma, C] & =\mathcal{Z}[C] W[\Sigma, C] .
\end{aligned}
$$

Infinitesimal versions of these transformations agree with the transformations that can be derived from [迆. Let us consider an infinitesimal surface $\delta \Sigma$ with the area element $\sigma^{\mu \nu}$. Assume that $M[C] \in \operatorname{Aut}(G)$ is an inner automorphism given by

$$
\begin{aligned}
M[C](g) & =\hat{P} \exp \left(\int_{C} \mu\right) g \hat{P} \exp \left(-\int_{C} \mu\right) \\
& =\hat{P} \exp \left(\int_{C} \mu_{\text {adjoint }}\right)(g), \quad \forall g \in G,
\end{aligned}
$$

where $\mu$ is a $\operatorname{Lie}(G)$-valued 1-form. From eq. (3.21) and

$$
W[\delta \Sigma] \approx 1+B_{\mu \nu} \sigma^{\mu \nu},
$$

one can find the transformation of the 2-form $B$ :

$$
\tilde{B}=B+d \zeta-\frac{1}{2}[\zeta, \zeta]-[\mu, \zeta]
$$

The transformation of $B$ corresponding to eqs.(3.4,3.5) reads

$$
\tilde{B}_{\mathrm{ab}}=B_{\mathrm{ab}}, \quad \tilde{B}_{\text {nonab }}=B_{\text {nonab }}-\rho,
$$

where $\rho$ is a $\operatorname{Lie}(G)$-valued 2 -form defined in

$$
R[\delta \Sigma] \approx 1+\rho_{\mu \nu} \sigma^{\mu \nu} .
$$

Eq. (3.25) agrees with the transformations that can be derived from 囵.

Unlike the gauge transformations (3.4)-(3.6), (3.15), the transformation (3.21) is not compatible with the composition rule (2.6). To find the correct transformation, $\mathcal{Z}[C]$ in eq. (3.21) should be 'smeared' over the surface $\Sigma$. We give an explicit formula for the gauge transformation of $V[\Sigma]$. It reads

$$
\tilde{V}[\Sigma]=\hat{P}_{\tau} \exp \left(\int_{\Sigma} i_{\mathcal{Z}\left[C_{P}\right]} M\left[C_{P}\right] v[P] M\left[C_{P}^{-1}\right] i_{\mathcal{Z}\left[C_{P}\right]^{-1}}\right) .
$$




\section{Comments}

- We found three kinds of gauge transformations of $M, V$ and $W$. These are $\Lambda[P]$ transformations (3.11), (3.15), $R[\Sigma]$-transformations (3.4)-(3.6) and $\mathcal{Z}[C]$-transformations (3.16), (3.21). Eq. (3.21) is valid only for infinitesimal surfaces and should be replaced by a 'smeared' version eq. (3.27).

- The ambiguity in surface-ordering necessitates the introduction of gauge transformations which compensate the ambiguity. Locally this amounts to the transformation eq. (3.25). The number of gauge degrees of freedom present in a NWS is enormous. Thus NWS may be relevant to a topological string theory describing topological sectors of the non-abelian string of [2].

- Infinitesimal version of eq. (2.6) can be derived from the composition rule for the natural transformation $K$ in figure 1 .

- We defined NWS on a local trivial patch. To define NWS globally one should cover the manifold with an atlas $\left\{U_{\alpha}\right\}$ and introduce $W_{\alpha}, V_{\alpha}, M_{\alpha}$ for each patch $U_{\alpha}$. As usual the quantities on the overlaps $U_{\alpha \beta}=U_{\alpha} \cap U_{\beta}$ are related by the gauge transformations. An analysis of global issues will be carried out elsewhere.

- We defined NWS with the disk topology. A generalization to higher-genus surfaces will be discussed elsewhere.

Note added. After submitting the original version of this paper to hep-th, the work [5 was brought to our attention. In [⿰] an equation similar to eq. (2.13) was taken as a definition of Wilson surface. The case considered in [5] corresponds, in our notation, to the $C$-independent $M[C]$. The surface-ordering ambiguities are absent in this case. For a list of miscellaneous work on non-abelian 2-form theories, see [6.

\section{Acknowledgments}

This work was supported by the DOE under grant No. DE-FG03-92ER40701, by the Research Foundation under NSF grant PHY-9722101 and by CRDF Award RP1-2108.

\section{References}

[1] L. Breen and W. Messing, Differential geometry of Gerbes, math.AG/0106083.

[2] A. Strominger, Open p-branes, Phys. Lett. B 383 (1996) 44 hep-th/9512059; O.J. Ganor, Six-dimensional tensionless strings in the large-N limit, Nucl. Phys. B 489 (1997) 95 hep-th/9605201.

[3] C. Teitelboim, Gauge invariance for extended objects, Phys. Lett. B 167 (1986) 63.

[4] M.B. Halpern, Field strength and dual variable formulations of gauge theory, Phys. Rev. D 19 (1979) 517;

I.Ya. Aref'eva, Non-abelian Stokes formula, Theor. Math. Phys. 43 (1980) 353;

P.M. Fishbane, S. Gasiorowicz and P. Kaus, Stokes' theorems for nonabelian fields, Phys. Rev. D 24 (1981) 2324; 
R.L. Karp, F. Mansouri and J.S. Rno, Product integral formalism and non-abelian Stokes theorem, J. Math. Phys. 40 (1999) 6033 hep-th/9910173;

Product integral representations of wilson lines and Wilson loops and non-abelian Stokes theorem, Turk. J. Phys. 24 (2000) 365 [hep-th/9903221].

[5] O. Alvarez, L.A. Ferreira and J. Sanchez Guillen, A new approach to integrable theories in any dimension, Nucl. Phys. B 529 (1998) 689 hep-th/9710147.

[6] P.G.O. Freund and R.I. Nepomechie, Unified geometry of antisymmetric tensor gauge fields and gravity, Nucl. Phys. B 199 (1982) 482;

P. Orland, Duality for nonabelian lattice fields, Nucl. Phys. B 163 (1980) 275:

D.Z. Freedman and P.K. Townsend, Antisymmetric tensor gauge theories and nonlinear sigma models, Nucl. Phys. B 177 (1981) 282,

A. Lahiri, Gauge transformations of the non-abelian two-form, hep-th/0107104 Local symmetries of the non-abelian two-form, hep-th/0109220. 